\title{
A PRODUCT INTEGRAL REPRESENTATION FOR A GRONWALL INEQUALITY
}

\author{
BURRELL W. HELTON
}

1. Introduction. This paper shows that if $G$ and $H$ are functions from $R \times R$ to $R$ such that $1-G \geqq c>0, G$ and $H$ are integrable and have bounded variation on $[a, b], f$ is bounded and $f(x) \leqq k$ $+(L R) \int_{a}^{x}(f H+f G)$ for $x \in[a, b]$ then

(1) if $G \geqq 0$ and $H \geqq 0$, then $f(x) \leqq k \prod_{a}^{x}(1+H)(1-G)^{-1}$ and $k \prod_{a}^{x}(1+H)(1-G)^{-1}$ is a solution of the inequality,

(2) if $1-|G| \geqq c>0$ and $f \geqq 0$, then $f(x) \leqq k \prod_{a}^{x}(1+|H|)(1-|G|)^{-1}$, and

(3) if $k \geqq 0$, the requirement $1-G \geqq c>0$ cannot be relaxed. Also a Gronwall-type inequality is stated and proved for functions $f, G$ and $H$ which have ranges in a normed ring.

The Main Theorem of Schmaedeke and Sell [3] is a special case of Theorem 4 of this paper. The linear function $J(f)$ defined by Herod [2] is more general than the function $J(f)=(L R) \int(f H+f G)$ defined above; however, there are linear functions $(L R) \int_{x}^{u}(f H+f G)$ which will satisfy the hypothesis of Theorem 4 but will not satisfy the hypothesis of Herod's theorem.

2. Definitions and preliminary theorems. For detailed definitions, see [1, p. 299]. All sum and product integrals (represented by the symbol $\left.\prod_{a}^{b} G\right)$ are subdivision-refinement-type limits of appropriate sums or products: $(L R) \int_{a}^{b}(f H+f G) \sim f(x) H(x, y)+f(y) G(x, y)$, $(m) \int_{a}^{b} G f \sim \frac{1}{2}[f(x)+f(y)] G(x, y), \prod_{a}^{b}(1+H)(1-G)^{-1} \sim[1+H(x, y)]$ $[1-G(x, y)]^{-1}$, etc. and it is understood that $a \leqq x<y \leqq b ; R$ is the set of real numbers, and $N$ is a ring which has a multiplicative element 1 and has a norm $|\cdot|$ with respect to which $N$ is complete and $|1|=1$; $f, u, v, G, H$ are functions from $R$ or $R \times R$ to $N$. $G \in O A^{\circ}$ on $[a, b]$ iff $\int_{a}^{b} G$ exists and $\int_{a}^{b}\left|G-\int G\right|=0 ; G \in O M^{\circ}$ on $[a, b]$ iff $\prod_{x}^{v}(1+G)$ exists for $a \leqq x<y \leqq b$ and $\int_{a}^{b}|(1+G)-\Pi(1+G)|=0 ; \quad G \in O L^{\circ}$ iff $\lim _{x \rightarrow p^{-}} G(x, p), \lim _{x \rightarrow p^{+}} G(p, x), \lim _{x, y \rightarrow p^{-}} G(x, y)$ and $\lim _{x, y \rightarrow p^{+}} G(p, x)$ exist for $p \in[a, b]$. The function $G$ is bounded on $[a, b]$ means there is a subdivision $\left\{x_{i}\right\}_{0}^{n}$ of $[a, b]$ and a number $M$ such that if $0<i \leqq n$ and $x_{i-1} \leqq x<y \leqq x_{i}$ then $|G(x, y)|<M$. A similar meaning is given to each statement such as $G>0$ on $[a, b],(1-G)^{-1}$ exists on $[a, b]$, etc.

Received by the editors May $5,1969$. 
THEOREM 1. Given: $f$ and $h$ are functions from $R$ to $N$ and $H, G$ and $B$ are functions from $R \times R$ to $N$ such that on $[a, b], h$ has bounded variation, $(1-G)^{-1}$ exists and is bounded, $d h(1-G)^{-1} \in O A^{\circ}, B=(1+H)$ $(1-G)^{-1}, B-1$ has bounded variation and $\prod_{x}^{y} B$ exists for $a \leqq x<y \leqq b$.

Conclusion. The following two statements are equivalent:

(1) $f(x) H(x, y)+f(y) G(x, y) \in O A^{\circ}$ and $f(x)=h(x)+(L R) \int_{a}^{x}(f H+f G)$ for $x \in[a, b]$; and

(2) if $a \leqq x<y \leqq b$, then $(L) \int_{a}^{y}|f(t)[B-\Pi B]|=0$ and $f(y)$ $=f(x) \prod_{x}^{v} B+(R) \int_{x}^{y} d h(1-G)^{-1} \prod_{i}^{y} B$.

This theorem is a special case of Theorem $5.1[1$, p. 310].

THEOREM 2. If $H$ and $G$ are functions from $R \times R$ to $N$ such that $H \in O L^{\circ}, G \in O A^{\circ}$ and $G$ has bounded variation on $[a, b]$, then $G H$ and $H G \in O A^{\circ}$ and $O M^{\circ}$ on $[a, b]$. Furthermore, if $H$ has bounded variation and $H \in O A^{\circ}$ on $[a, b]$ then $\int_{a}^{b} G H=\sum_{x \in S} G\left(x^{-}, x\right) H\left(x^{-}, x\right)$ $+G\left(x, x^{+}\right) H\left(x, x^{+}\right)$, where $S$ is the subset of $[a, b]$ such that $x \in S$ iff $G$ has a discontinuity at $x, G\left(a^{-}, a\right)=0$ and $G\left(b, b^{+}\right)=0$.

The proof of this theorem is given in $\$ 4$.

If $f, H, G$ are functions such that on $[a, b], f, H, G$ have bounded variation, $H \in O A^{\circ}, G \in O A^{\circ}$, and $(1-G)^{-1}$ exists and is bounded, then $(1-G)^{-1} \in O L^{\circ}$ on $[a, b]$ and it follows from Theorem 2 that $d f(1-G)^{-1}, H(1-G)^{-1}, f H, \quad(1+H)(1-G)^{-1}-1=(H+G)(1-G)^{-1}$ $\in O A^{\circ}$ and $O M^{\circ}$ on $[a, b]$.

\section{The principal results.}

TheOREM 3. $H$ and $G$ are functions of bounded variation from $R \times R$ to $R, c \in R, H \in O A^{\circ}, G \in O A^{\circ}, H \geqq 0, G \geqq 0$ and $1-G \geqq c>0$ on $[a, b]$ and $u$ is a function from $R$ to $R$ such that $u$ is bounded above on $[a, b]$, $(L R) \int_{a}^{b}(u H+u G)$ exists and $u(x) \leqq \int_{a}^{x}(u H+u G)$ for $x \in[a, b]$.

Conclusion. If $x \in[a, b]$, then $u(x) \leqq 0$.

Proof. Assume the conclusion is false and let $S$ be the subset of $[a, b]$ such that $x \in S$ iff $u(x)>0$; then $S$ is nonempty and has a greatest lower bound $p$. Since

$$
\begin{aligned}
u(p) & \leqq(L R) \int_{a}^{p^{-}}(u H+u G)+(L R) \int_{p^{-}}^{p}(u H+u G) \\
& \leqq(L R) \int_{p^{-}}^{p}(u H+u G) \leqq u(p) G\left(p^{-}, p\right),
\end{aligned}
$$

then $u(p)\left[1-G\left(p^{-}, p\right)\right] \leqq 0$ and $u(p) \leqq 0$; furthermore, $p<b$.

Since $H$ and $G$ have bounded variation and since $G\left(p, p^{+}\right)<1$, then 
there is a number $y, p<y \leqq b$, such that $\int_{p^{+}}^{y} H+\int_{p}^{y} G<\frac{1}{2}\left[G\left(p, p^{+}\right)+1\right]$. Let $M$ be the least upper bound for $u$ on $[p, y]$; then there is a number $z \in[p, y]$ such that $u(z)>\frac{1}{2} M\left[G\left(p, p^{+}\right)+1\right]$. Hence,

$$
\begin{aligned}
u(z) & \leqq(L R) \int_{a}^{z}(u H+u G)=(L R)\left(\int_{a}^{p}+\int_{p}^{p^{+}}+\int_{p^{+}}^{z}\right)(u H+u G) \\
& \leqq u(p) H\left(p, p^{+}\right)+M G\left(p, p^{+}\right)+\int_{p^{+}}^{z}(M H+M G) \\
& \leqq M\left(\int_{p^{+}}^{z} H+\int_{p}^{z} G\right)<\frac{1}{2} M\left[G\left(p, p^{+}\right)+1\right]<u(z) .
\end{aligned}
$$

This contradiction proves that $u(x) \leqq 0$ for $x \in[a, b]$.

THEOREM 4. Given: $H$ and $G$ are functions of bounded variation from $R \times R$ to $R, c \in R, H \in O A^{\circ}, G \in O A^{\circ}$ and $1-G \geqq c>0$ on $[a, b]$ and $f$ is a function from $R$ to $R$ such that $f$ is bounded above on $[a, b],(L R) \int_{a}^{b}$ $(f H+f G)$ exists, $k$ is a number and $f(x) \leqq k+(L R) \int_{a}^{x}(f H+f G)$ for $x \in[a, b]$.

Conclusion. (1) If $H \geqq 0$ and $G \geqq 0$ on $[a, b]$, then

$$
f(x) \leqq k \prod_{a}^{x}(1+H)(1-G)^{-1}=k \prod_{a}^{x}(1+H) / \prod_{a}^{x}(1-G)
$$

for $a \leqq x \leqq b$. Furthermore, the function $f(x)=k \prod_{a}^{x-}(1+H)(1-G)^{-1}$ is a solution to the inequality.

(2) If $c \in R, 1-|G| \geqq c>0,(L R) \int_{a}^{b}(f|H|+f|G|)$ exists and $f \geqq 0$ on $[a, b]$, then

for $a \leqq x \leqq b$.

$$
\begin{aligned}
f(x) & \leqq k \prod_{a}^{x}(1+|H|)(1-|G|)^{-1} \\
& =k \prod_{a}^{x}(1+|H|) / \prod_{a}^{x}(1-|G|)
\end{aligned}
$$

Proof OF PART 1 . Suppose $H \geqq 0$ and $G \geqq 0$ on $[a, b]$. Since $(1-G)^{-1}$ exists and is bounded on $[a, b]$, it follows from Theorem 2 that $(1+H)(1-G)^{-1}-1 \in O M^{\circ}$ and has bounded variation on $[a, b]$. Let $v$ be the function such that $v(x)=k \prod_{a}^{x}(1+H)(1-G)^{-1}$ for $x \in[a, b]$; then $v$ is bounded on $[a, b]$. It follows from Theorem $1(2 \rightarrow 1)$ that $v(x)=k+(L R) \int_{a}^{x}(v H+v G)$. Let $u=f-v$; then, for $x \in[a, b]$,

$$
u(x) \leqq(L R) \int_{a}^{x}[(f-v) H+(f-v) G]=(L R) \int_{a}^{x}(u H+u G) .
$$


Since $f$ is bounded above, then $u$ is bounded above on $[a, b]$ and, from Theorem 3, $u(x) \leqq 0$; hence, $f(x) \leqq v(x)=k \prod_{a}^{x}(1+H)(1-G)^{-1}$ $=k \prod_{a}^{x}(1+H) / \prod_{a}^{x}(1-G)$, since $\prod_{a}^{x}(1-G) \neq 0$. The second half of part 1 follows because $k \prod_{a}^{x}(1+H)(1-G)^{-1}$ is a solution of the equation $f(x)=k+(L R) \int_{a}^{x}(f H+f G)$.

PROOF OF PART 2. Suppose $1-|G| \geqq c>0,(L R) \int_{a}^{b}(f|H|+f|G|)$ exists and $f \geqq 0$ on $[a, b]$. Since $|H| \in O A^{\circ}$ and $|G| \in O A^{\circ}$ and

$$
f(x) \leqq k+(L R) \int_{a}^{x}(f H+f G) \leqq k+(L R) \int_{a}^{x}(f|H|+f|G|)
$$

for $x \in[a, b]$, the desired inequality follows from part 1 above. Note that if $f$ is quasicontinuous, it follows from Theorem 2 that $(L R) \int_{a}^{b}$ $(f|H|+f|G|)$ exists.

TheOREM 5. If $H$ and $G$ are functions from $R \times R$ to $R, G\left(b^{-}, b\right) \geqq 1$, and $M$ and $k$ are nonnegative numbers, then there is a function $f$ such that $f(x) \leqq k+(L R) \int_{a}^{x}(f H+f G)$ for $x \in[a, b]$ and $f(b)>k M$.

Proof. Let $f$ be the function such that $f=0$ on $[a, b)$ and $f(b)>k M$. Then

$$
f(b) \leqq f(b) G\left(b^{-}, b\right)=(L R) \int_{a}^{b}(f H+f G) \leqq k+(L R) \int_{a}^{b}(f H+f G),
$$

and, if $x \in[a, b), f(x)=0 \leqq k+(L R) \int_{a}^{x}(f H+f G)$.

THEOREM 6. If $M, k$ and $c$ are numbers such that $M>0$ and $c>0$ and $H$ and $G$ are functions from $R \times R$ to $R$ such that $G\left(b^{-}, b\right)>1$ and on $[a, b] H$ and $G$ have bounded variation, $H \in O A^{\circ}, G \in O A^{\circ}$ and $|1-G|$ $>c$, then there is a function $f$ from $R$ to $R$ such that $f(b)>|k| M$ and $f(x) \leqq k+(L R) \int_{a}^{x}(f H+f G)$ for $x \in[a, b]$.

Proof. Let $f$ be a function such that $f(x)=k \coprod_{a}^{x}(1+H)(1-G)^{-1}$ for $x \in[a, b)$ and $f(b)$ is a number such that $f(b)>|k| M$ and $f(b)\left[G\left(b^{-}, b\right)-1\right]+\left[(L) \int_{a}^{b} f H+(R) \int_{a}^{b-} f G+k\right]>0$. From Theorem $4, f$ is a solution on $[a, b)$. Also,

$$
\begin{aligned}
(L R) \int_{a}^{b}(f H+f G)= & (L) \int_{a}^{b} f H+(R) \int_{a}^{b^{-}} f G+f(b)\left[G\left(b^{-}, b\right)-1\right] \\
& +f(b)+k-k>f(b)-k
\end{aligned}
$$

therefore, $f$ is a solution on $[a, b]$.

In the following theorem, $A$ and $B$ denote the functions $B$ $=(1+H)(1-G)^{-1}$ and $A(p, q)=\prod_{p}^{q} B ; P$ denotes a bound for $A$ on $[a, b] ; Q(x, y)=G(x, y)[1-G(x, y)]^{-1} P$ and $M(a, x)$ is the sum of the 
magnitudes of the discontinuities of $Q$ on $[a, b]$. Note that $N$ is a normed ring and that the inequalities $\left|G\left(x^{-}, x\right)\right|>1$ and $\left|G\left(x, x^{+}\right)\right|$ $>1$ are permitted.

Theorem 7. Given: $k>0, f$ is a function from $R$ to $N, G$ and $H$ are functions from $R \times R$ to $N$ such that on $[a, b] f, G$ and $H$ have bounded variation, $G \in O A^{\circ}, H \in O A^{\circ}$, and $(1-G)^{-1}$ exists and is bounded.

Conclusion. If $\left|f(x)-(L R) \int_{a}^{x}(f H+f G)\right|<k$ for $a \leqq x \leqq b$, then

$$
|f(y)| \leqq k\left[1+V_{a}^{y} A+2 M(a, y)\right]
$$

for $a \leqq y \leqq b$.

ProOF. Let $h$ be the function such that $h(y)=f(y)-(L R) \int_{a}^{y}(f H+f G)$ for $a \leqq y \leqq b$. Since $f, H$ and $G$ have bounded variation, then $h$ has has bounded variation. The function $B-1=(1+H)(1-G)^{-1}-1$ $=(H+G)(1-G)^{-1}$ has bounded variation and, from Theorem 2, $d h(1-G)^{-1} \in O A^{\circ}, f(x) H(x, y)+f(y) G(x, y) \in O A^{\circ}, B-1 \in O M^{\circ}$, and $\prod_{x}^{y} B$ exists for $a \leqq x<y \leqq b$. Since $f(y)=h(y)+(L R) \int_{a}^{y}(f H+f G)$, the hypothesis of Theorem $1(1 \rightarrow 2)$ is satisfied and for $a \leqq y \leqq b$

$$
\begin{aligned}
f(y) & =f(a) A(a, y)+(R) \int_{a}^{y} d h(1-G)^{-1} A(t, y) \\
& =f(a) A(a, y)+(R) \int_{a}^{y} d h\left[1+G(1-G)^{-1}\right] A(t, y) \\
& =f(a) A(a, y)+(R) \int_{a}^{y} d h A(t, y)+(R) \int_{a}^{y} d h G(1-G)^{-1} A(t, y),
\end{aligned}
$$

and

$$
\begin{aligned}
f(a) A(a, y) & +(R) \int_{a}^{y} d h A(t, y) \\
= & f(a) A(a, y)+\left.h(t) A(t, y)\right|_{a} ^{y}-(L) \int_{a}^{y} h d A(t, y) \\
= & f(a) A(a, y)+h(y) A(y, y)-h(a) A(a, y)-(L) \int_{a}^{y} h d A(t, y) \\
= & h(y)-(L) \int_{a}^{y} h d A(t, y) .
\end{aligned}
$$

From Theorem 2, it follows that $\left|(R) \int_{a}^{y} d h\left[G(1-G)^{-1} A(t, y)\right]\right|$ $\leqq 2 k M(a, y)$. Hence, 


$$
\begin{aligned}
|f(y)| & \leqq h(y)-(L) \int_{a}^{y} h d A(t, y)+(R) \int_{a}^{y} d h\left[G(1-G)^{-1} A(t, y)\right] \mid \\
& \leqq k+k V_{a}^{y} A+2 k M(a, y)=k\left[1+V_{a}^{y} A+2 M(a, y)\right] .
\end{aligned}
$$

If $H(x, y)=G(x, y)=\frac{1}{2}[g(y)-g(x)]$, then $(m) \int f d g=(L R) \int(f H+f G)$, $(m) \int f d g$ is a special case of $(L R) \int(f H+f G)$, and Schmaedeke and Sell's Main Theorem [3, p. 1219] is a special case of Theorem 4. Similarly, $(R) \int f d g$, $(L) \int f d g$ and the Riemann-Stieltjes integral are special cases. If $f$ is left or right continuous on $[a, b]$, then $(D) \int f d g$ $=(R) \int f h$ or $(D) \int f d g=(L) \int f G$, respectively, where $(D) \int f d g$ is the Dushkin integral [3, p. 1218]. Herod's linear function $J(f)[2$, p. 570] is more general than the function $J(f)(x, y)=(L R) \int_{x}^{y}(f H+f G)$; however, the results of Theorem 4 are better than Herod's results in the sense that Theorem 4 permits $f$ to have unbounded variation and permits $(L R) \int_{x}^{y}(f H+f G)$ to have discontinuities greater than 1 . Note that the function $\prod_{x}^{y}(1+H)(1-G)^{-1}$ defined in Theorem 4 satisfies each of the properties listed by Herod for the function $m(x, y): m(x, y)$ $\geqq 1, m(x, y) m(y, z)=m(x, z)$ for $x<y<z$, and $m(0, x)=1+J[m(0, \cdot)]$ $(0, x)$.

4. Proof of Theorem 2. In this section Theorem 2 and a necessary lemma are proved.

Lemma. Given: $H$ is a function from $R \times R$ to $N, H \in O L^{\circ}$ on $[a, b]$, $e>0$, and $S^{-}$and $S^{+}$are subsets of $[a, b]$ such that $p \in S^{-}$iff

$$
\left|\lim _{x, y \rightarrow p^{-}} H(x, y)-H\left(p^{-}, p\right)\right| \geqq e
$$

and

$$
p \in S^{+} \quad \text { iff }\left|\lim _{x, y \rightarrow p^{+}} H(x, y)-H\left(p, p^{+}\right)\right| \geqq e .
$$

Conclusion. (1) $S^{-}$and $S^{+}$are finite sets and (2) there is a subdivision $\left\{x_{i}\right\}_{0}^{n}$ of $[a, b]$ such that $H$ is bounded on $\left[x_{i-1}, x_{i}\right]$ for $i$ $=1,2,3, \cdots, n$.

Proof. Suppose $S^{-}$is an infinite set; then $S^{-}$has an accumulation point $q \in[a, b]$ and there is a subset $\left\{p_{n}\right\}_{1}^{\infty}$ of $S^{-}$and a sequence $\left\{x_{n}, y_{n}\right\}_{1}^{\infty}$ of number pairs such that $p_{n} \rightarrow q^{-}$and $x_{n}, y_{n} \rightarrow q^{-}$(or $p_{n} \rightarrow q^{+}$ and $\left.x_{n}, y_{n} \rightarrow q^{+}\right)$and such that $\left|H\left(x_{n}, y_{n}\right)-H\left(p_{n}^{-}, p_{n}\right)\right| \geqq e$ for $n$ $=1,2,3, \cdots$. Since $H \in O L^{\circ}$,

$$
\lim _{n \rightarrow \infty} H\left(x_{n}, y_{n}\right)=\lim _{n \rightarrow \infty} H\left(\overline{p_{n}}, p_{n}\right)
$$

and 


$$
0=\lim _{n \rightarrow \infty}\left|\left[H\left(x_{n}, y_{n}\right)-H\left(\overline{p_{n}}, p_{n}\right)\right]\right| \geqq e .
$$

Similarly, $S^{+}$is a finite set.

Since $H \in O L^{\circ}$, then $H$ is bounded in a neighborhood of each point of $[a, b]$. The covering theorem assures that there is a subdivision which has the desired property.

Proof of theorem 2. Let $\epsilon>0$ and let $M$ be the number and $A, B, C, D, E, T_{i}$ be the number sets defined as follows:

(1) $A=\left\{a_{i}\right\}_{0}^{r}$ is a subdivision of $[a, b]$ and $M$ is a number such that if $0<i \leqq r$ and $a_{i-1} \leqq x<y \leqq a_{i}$ then $|H(x, y)|<M$.

(2) $B=\left\{b_{i}\right\}_{1}^{s}$ is the subset of $[a, b]$ such that $p \in B$ iff

$$
\left|\lim _{x, y \rightarrow p^{+}} H(x, y)-H\left(p, p^{+}\right)\right| \geqq \epsilon /\left(8 V_{a}^{b} G\right)
$$

or

$$
\left|\lim _{x, y \rightarrow p^{-}} H(x, y)-H\left(p^{-}, p\right)\right| \geqq \epsilon /\left(8 V_{a}^{b} G\right) .
$$

(3) $C=\left\{c_{i}\right\}_{1}^{s}$ and $D=\left\{d_{i}\right\}_{1}^{s}$ are subsets of $[a, b]$ such that $c_{i}<b_{i}<d_{i}$ for $i=1,2, \cdots, s$ and

$$
\sum_{1}^{s}\left(V_{c_{i}}^{b_{i}^{-}} G+V_{b_{i}^{+}}^{d_{i}} G\right)<\epsilon / 8 M
$$

and $\left|H\left(x, b_{i}\right)-H\left(y, b_{i}\right)\right|,\left|H\left(b_{i}, x\right)-H\left(b_{i}, y\right)\right|$ and $|H(x, y)-H(p, q)|$ are less than $\epsilon /\left(8 V_{a}^{b} G\right)$ whenever $x, y, p, q \in\left[c_{i}, b_{i}\right)$ or $x, y, p, q \in\left(b_{i}, d_{i}\right]$.

(4) $T_{i}=\left\{t_{i j}\right\}_{j}$ for $i=1,2, \cdots, s$ is a subdivision of $\left[d_{i}, c_{i+1}\right]$ such that, if $t_{i j} \leqq x<y \leqq t_{i, j+1}$ and $t_{i j} \leqq p<q \leqq t_{i, j+1}$, then $|H(x, y)-H(p, q)|$ $<\epsilon /\left(8 V_{a}^{b} G\right)$.

(5) $E=\left\{z_{i}\right\}_{1}^{m}$ is a subdivision of $[a, b]$ such that if $D^{\prime}=\left\{y_{i j}\right\}_{i, j}$ is a refinement of $E$, then

$$
\sum_{i}\left|G_{i}-\sum_{j} G_{i j}\right|<\epsilon / 8 M
$$

where $G_{i}=G\left(z_{i-1}, z_{i}\right)$ and $G_{i j}=G\left(y_{i, j-1}, y_{i j}\right)$ and $z_{i-1} \leqq y_{i, j-1}<y_{i j} \leqq z_{i}$. Similar abbreviated notations are used in the following manipulations.

Let $D^{\prime}=\left\{x_{i j}\right\}_{i, j}$ be a refinement of the subdivision $K=$ $A \cup B \cup C \cup D \cup E \bigcup_{1}^{s} T_{i}=\left\{x_{i}\right\}_{i}$. In the following, $\sum_{i}$ depends on $K$; $\sum_{j} G_{i j}$ depends on $D^{\prime}$ and $\left[x_{i-1}, x_{i}\right] ; i \in Q$ iff $x_{i} \in B$. 


$$
\begin{aligned}
& \sum_{i}\left|\sum_{j} H_{i j} G_{i j}-H_{i} G_{i}\right| \\
&=\sum_{i}\left|H_{i}\left(\sum_{j} G_{i j}-G_{i}\right)+\sum_{j}\left(H_{i j}-H_{i}\right) G_{i j}\right| \\
& \leqq \sum_{i}\left|H_{i}\right| \cdot\left|\sum_{j} G_{i j}-G_{i}\right|+\sum_{i \in Q} \sum_{j}\left|\left(H_{i j}-H_{i}\right) G_{i j}\right| \\
&+\sum_{i \nsubseteq Q} \sum_{j}\left|\left(H_{i j}-H_{i}\right)\right|\left|G_{i j}\right| \\
&< M(\epsilon / 8 M)+\sum_{i \in Q} \sum_{j} b_{i j}\left|H_{i j}-H_{i}\right|\left|G_{i j}\right| \\
&+\sum_{i \in Q} \sum_{j} a_{i j}\left|H_{i j}-H_{i}\right|\left|G_{i j}\right|+\left(\epsilon / 8 V_{a}^{b} G\right) V_{a}^{b} G \\
&< \epsilon / 8+\left(\epsilon / 8 V_{a}^{b} G\right) V_{a}^{b} G+2 M \sum_{i}\left(V_{c ;}^{b_{i}^{-}} G+V_{b_{i}^{+}}^{d_{i}} G\right)+\epsilon / 8<\epsilon,
\end{aligned}
$$

where $a_{i j}=1$ and $b_{i j}=0$ provided $i \in Q$ and $x_{i j}$ is the largest element of $D^{\prime}$ such that $x_{i j}<x_{i}$ or $x_{i j}$ is the smallest element of $D^{\prime}$ such that $x_{i j}>x_{i}$; otherwise, $a_{i j}=0$ and $b_{i j}=1$. Hence, $H G \in O A^{\circ}$ and, similarly, $G H \in O A^{\circ}$. It follows from Theorem $3.4[1$, p. 301] that $H G$ and GHEOM .

Suppose $H$ has bounded variation and $H \in O A^{\circ}$ on $[a, b]$ and let $g$ and $h$ be the functions such that $g(x)=G(a, x)$ and $h(x)=H(a, x)$; then $g$ and $h$ are quasicontinuous and it follows from Theorem 3.1 $[1$, p. 300] that

$$
\begin{aligned}
\int_{a}^{b} G H= & \int_{a}^{b}\left(\int G\right)\left(\int H\right)=\int_{a}^{b} d g d h \\
= & \sum_{x \in S}\left\{\left[g(x)-g\left(x^{-}\right)\right]\left[h(x)-h\left(x^{-}\right)\right]\right. \\
& \left.+\left[g\left(x^{+}\right)-g(x)\right]\left[h\left(x^{+}\right)-h(x)\right]\right\} .
\end{aligned}
$$

\section{BiBLIOGRAPHY}

1. B. W. Helton, Integral equations and product integrals, Pacific J. Math. 16 (1966), 297-322.

2. J. V. Herod, A Gronwall inequality for linear Stieltjes integrals, Notices Amer. Math. Soc. 16 (1969), 570.

3. W. W. Schmaedeke and G. R. Sell, The Gronwall inequality for modified Stieltjes integrals, Proc. Amer. Math. Soc. 19 (1968), 1217-1222.

Southwest Texas State University 